\title{
Chronic exposure to cadmium induces a malignant transformation of benign prostate epithelial cells
}

\author{
Balaji Chandrasekaran', Nisha R. Dahiya', Ashish Tyagi', Venkatesh Kolluru', Uttara Saran', Becca V. Baby', \\ J. Christopher States ${ }^{2}$, Ahmed Q. Haddad', Murali K. Ankem' and Chendil Damodaran'
}

\begin{abstract}
Epidemiological evidence suggests that cadmium (Cd) is one of the causative factors of prostate cancer, but the effect of $\mathrm{Cd}$ on benign prostatic hyperplasia (BPH) remains unclear. This study aimed to determine whether $\mathrm{Cd}$ exposure could malignantly transform BPH1 cells and, if so, to dissect the mechanism of action. We deciphered the molecular signaling responsible for $\mathrm{BPH} 1$ transformation via RNA-sequencing and determined that $\mathrm{Cd}$ induced the expression of zinc finger of the cerebellum 2 (ZIC2) in BPH1 cells. We noted $\mathrm{Cd}$ exposure increased ZIC2 expression in the $\mathrm{Cd}$ transformed $\mathrm{BPH} 1$ cells that in turn promoted anchorage-independent spheroids and increased expression of stem cell drivers, indicating their role in stem cell renewal. Subsequent silencing of ZIC2 expression in transformed cells inhibited spheroid formation, stem cell marker expression, and tumor growth in nude mice. At the molecular level, ZIC2 interacts with the glioma-associated oncogene family (GLI) zinc finger 1 (GLI1), which activates prosurvival factors (nuclear factor NFkB, B-cell lymphoma-2 (Bcl2), as well as an X-linked inhibitor of apoptosis protein (XIAP)) signaling in $\mathrm{Cd}$-exposed BPH1 cells. Conversely, overexpression of ZIC2 in BPH1 cells caused spheroid formation confirming the oncogenic function of ZIC2. ZIC2 activation and GLI1 signaling induction by $\mathrm{Cd}$ exposure in primary BPH cells confirmed the clinical significance of this oncogenic function. Finally, human BPH specimens had increased ZIC2 versus adjacent healthy tissues. Thus, we report direct evidence that $\mathrm{Cd}$ exposure induces malignant transformation of $\mathrm{BPH}$ via activation of ZIC2 and GLI1 signaling.
\end{abstract}

\section{Introduction}

Benign prostatic hyperplasia (BPH) is a chronic urological disorder characterized by noncancerous enlargement of the prostate gland ${ }^{1,2}$. Both BPH and prostate cancer $(\mathrm{CaP})$ share similar etiology and pathophysiological factors $^{3-5}$. Although, $\mathrm{BPH}$ to $\mathrm{CaP}$ conversion is controversial, the published evidence suggests that $\mathrm{BPH}$ patients have a two to threefold increased risk for $\mathrm{CaP}$ and a two to eightfold increased risk for CaP-associated mortality $^{6}$. More recently, a comprehensive review reported an association between $\mathrm{BPH}$ and $\mathrm{CaP}$, suggesting that $\mathrm{BPH}$ is a risk factor for $\mathrm{CaP}^{7}$. While the possibility exists that $\mathrm{BPH}$ patients have an increased risk of

Correspondence: Chendil Damodaran (chendil.damodaran@louisville.edu)

'Department of Urology, University of Louisville, Louisville, KY, USA

${ }^{2}$ Pharmacology and Toxicology, University of Louisville, Louisville, KY, USA

These authors contributed equally: Balaji Chandrasekaran, Nisha Dahiya developing $\mathrm{CaP}$ and $\mathrm{CaP}$-associated mortality, it remains unclear how and why BPH patients are at risk for developing CaP.

Cadmium (Cd) is a known metal carcinogen and is one of the most abundant occupational and environmental pollutants found in air, soil, water, dietary products, and tobacco smoke ${ }^{8}$. Epidemiological studies have reported $\mathrm{Cd}$ could be a potent prostate carcinogen because it is found in significantly higher levels in tissues and plasma of $\mathrm{CaP}$ patients than healthy controls ${ }^{9}$. $\mathrm{Cd}$ is an endocrine disruptor in experimental models ${ }^{10}$, supporting the hypothesis that this metal carcinogen can potentially induce the development of hormone-dependent tumors in humans, including that of the breast and uterus ${ }^{11,12}$. However, its molecular effects on malignant transformation of BPH cells remain elusive. 
Deregulation of transcription factors, such as the zinc finger of cerebellum 2 (ZIC2) has been linked to heavy metal exposure and metal-induced carcinogenesis ${ }^{13}$. ZIC2 plays a regulatory function in pluripotent and self-renewal of cancer stem cells (CSCs) $)^{14,15}$ and is also highly expressed and involved in tumorigenesis of various cancer types, including $\mathrm{CaP}^{14-16}$. As a zinc finger transcriptional factor, ZIC2 binds with the zinc finger domains of other protein families, including glioma-associated oncogene (GLI) in either a synergistic or antagonistic manner ${ }^{17,18}$. GLI proteins are also the downstream targets of the Sonic hedgehog (Shh) pathway that are an important therapeutic target for $\mathrm{CaP}$ treatment. Several studies have demonstrated growth arrest in $\mathrm{CaP}$ cell lines and xenografted tumors in mice ${ }^{19,20}$ following the inhibition of Shh signaling. Shh-associated secretory protein binds and inactivates patched1 (PTCH1), resulting in the release of smoothened protein and activation of GLI1, GLI2, and GLI3 receptors. GLI1 predominantly functions as a transcription activator, while GLI2 and GLI3 function as either an activator or repressor ${ }^{21}$. GLI1 activation initiates the expression of downstream target genes involved in proliferation (cyclin D1), survival (B-cell lymphoma-2, $\mathrm{Bcl} 2$ ), metastasis (Snail), and stem cell activation (Nanog and SOX2) ${ }^{22}$. Activated GLI1 and GLI2 proteins can also directly promote the expression of a group of genes involved in the process of epithelial mesenchymal transition $(\mathrm{EMT})^{23}$.

Here, for the first time, we report direct evidence that $\mathrm{Cd}$ exposure induces malignant transformation of $\mathrm{BPH} 1$ cells that exhibit an aggressive phenotype similar to that of $\mathrm{CaP}$. In addition, our results suggest that chronic exposure of $\mathrm{Cd}$ to BPH1 cells was responsible for stem cell renewal, proliferation, and tumorigenesis of the transformed cells.

\section{Materials and methods \\ Cell lines and reagents}

The BPH1 cell line was a kind gift from Dr. Simon W. Hayward (Northshore Research institute University of Chicago, Pritzker School of Medicine). The cells were authenticated by Genetical Cell Line Testing (Burligton, NC, USA). The cells were treated with $10 \mu \mathrm{M}$ Cd (Sigma, Dallas, TX, USA) for 1 year and transformed into a malignant phenotype. The transformed cells were named Cd-transformed BPH1 (CTBPH1). Human normal prostate epithelial cells (RWPE-1) were purchased from the American Type Culture Collection (Manassas, VA, USA). BPH1 and CTBPH1 cells were cultured in RPMI medium supplemented with $10 \%$ fetal bovine serum, and $1 \%$ antibiotic and antimycotic solution in a humidified atmosphere of $5 \% \mathrm{CO}_{2}$ at $37^{\circ} \mathrm{C}$ in an incubator. The RWPE-1 cells were cultured in keratinocyte serum-free medium containing l-glutamine, Epidermal Growth Factor (EGF) and bovine pituitary extract (BPE).

\section{Cell viability assays}

Cell viability assays were performed using the trypan blue exclusion method or the 3-(4, 5-dimethylthiazol-2yl)-2, 5-diphenyltetrazolium-bromide (MTT) assay as described previously ${ }^{24}$. BPH1 cells following exposure to $10 \mu \mathrm{M} \mathrm{Cd}$ for 24,48 , and $72 \mathrm{~h}$.

\section{Soft agar colony formation assay}

Colony formation assays were performed on $\mathrm{Cd}$-exposed BPH1 and CTBPH1 cells to monitor anchorage-independent growth via the CytoSelectTM 96-well in vitro tumor sensitivity assay kit (Cell Biolabs, Inc., San Diego, CA, USA) ${ }^{25}$. A group of at least 50 or more cells were considered as a colony and those colonies were counted.

\section{Migration and invasion assays}

Invasion assays were performed on $\mathrm{BPH} 1,6$-month $\mathrm{Cd}$ exposed BPH1, CTBPH1 (12-month Cd-transformed cells), $\mathrm{ZIC}^{+}$, and $\mathrm{ZIC} 2^{-}$using Boyden chambers equipped with polyethylene terephthalate membranes with $8-\mu$ m pores (BD Biosciences) as described previously ${ }^{26}$. Similarly, migration assays were performed for $\mathrm{Cd}$-exposed $\mathrm{BPH} 1$ cells in sixwell plates as described previously ${ }^{24}$.

\section{Sphere formation assay}

Spheroids of $\mathrm{ZIC}^{+}$and $\mathrm{ZIC} 2^{-}$cells were generated by forced floating method using 96-well round-bottom Ultra Low Attachment plates (Corning ${ }^{\circledR}$, New York, USA). Single-cell suspension of $\mathrm{ZIC}^{+}$and $\mathrm{ZIC}^{-}$cells at a density of $2 \times 10^{3}$ cells in $200 \mu \mathrm{l}$ of respective culture media supplemented with Matrigel ${ }^{\mathrm{TM}}$ (354254, Corning) was loaded into each well. Then, the morphology of the spheroids, cell growth were characterized over a 7-day culture period in triplicate.

\section{Overexpression and siRNA transfection}

BPH1 cells were seeded in six-well plates at a density of $3 \times 10^{5}$ cells/well. After $24 \mathrm{~h}$, the cells were transiently transfected with short-interfering RNA (siRNA) specific for ZIC2 or GLI1, or control siRNA, or an overexpression plasmid specific for ZIC2 for $48 \mathrm{~h}$. Lipofectamine ${ }^{\circledR} 2000$ was used as a transfection reagent. After $48 \mathrm{~h}$, lysates were prepared and western blotting was performed.

\section{Immunoprecipitation (IP) and western blot}

$\mathrm{BPH} 1$, transforming cells $(2,4,6,8,10,12,14$, and 16 months), and CTBPH1 cells were seeded in six-well plates and incubated for $24 \mathrm{~h}$. Cell lysates were prepared, and western blotting was performed using specific antibodies against ZIC2 (ab150404), GLI1 (ab151796), Shh (ab53281), NANOG (cell signaling \#3608), ZIC2 immunohistochemistry (Sigma AU35821), CD44 (cell signaling \#5604), Poly(ADP-ribose) polymerase (PARP) (Cell signaling \#9542), NFkB-p65 (Cell signaling \#8242), Bcl2 
(Cell signaling \#2872), $\mathrm{Bcl}_{\mathrm{xL}}$ (Santa curtz 7B2.5), X-linked inhibitor of apoptosis protein (XIAP; Cell signaling \#2045), cleaved caspase-3 (Novus Biologicals NB10056112), PTCH1 (ab53281), NOTCH1 (Cell signaling \#3608), SOX2 (Cell signaling \#3579), E-cadherin (Cell signaling \#3195), N-cadherin (Cell signaling \#13116), Slug (Cell signaling \#9585), and $\beta$-catenin (Cell signaling \#8480). Protein-antibody complexes were visualized using enhanced chemiluminescence as previously descri$\operatorname{bed}^{25}$. For immunoprecipitation experiments, the protein samples were immunoprecipitated with ZIC2 antibody at $4{ }^{\circ} \mathrm{C}$ under agitation overnight following protein extraction using radioimmunoprecipitation assay (RIPA) buffer; the immunoprecipitated protein was then pulled down with protein A-agarose beads (Thermo Fisher Scientific, Rockford, IL) at $4{ }^{\circ} \mathrm{C}$ under rotary agitation for $3 \mathrm{~h}$. Immunoprecipitates were washed three times with RIPA buffer. After centrifugation, the pellets were resuspended in sample buffer and heated for $5 \mathrm{~min}$ at $95^{\circ} \mathrm{C}$ for sodium dodecyl sulfate-polyacrylamide gel electrophoresis followed by immunoblot analysis.

\section{Real-time Quantitative PCR (RT-qPCR)}

Total RNA was extracted from BPH1, Cd-exposed BPH1 (24, 48 and $72 \mathrm{~h}$ ), CTBPH1, and ZIC2 siRNAtreated CTBPH1 cells and RT-qPCR performed for ZIC2, SOX2, Nanog, CD44, and Notch1 expressions as described previously ${ }^{27}$.

Immunofluorescence and immunohistochemistry analysis

Immunofluorescence for ZIC2 and GLI1 expression was performed on Cd-exposed BPH1 and CTBPH1 cells, as described previously ${ }^{28}$. BPH tissue microarray and xenograft tumor tissues were examined for ZIC2, GLI1, and p65 expression using immunohistochemistry analysis.

\section{Cell sorting of $\mathrm{ZIC2}^{+}$and $\mathrm{ZIC2}^{-}$cells}

Cd-exposed BPH1 or CTBPH1 $\left(1 \times 10^{7}\right)$ cells were incubated with $2.0 \mu \mathrm{l}$ of fluorochrome-conjugated ZIC2 antibody before being sorted using fluorescence-activated cell sorting (FACS). Cells were analyzed on a Cytopeia Influx FACS using Spigot. Cell subpopulations were separated based on the surface antibody labeling and collected by discriminatory gating. After selecting for $\mathrm{ZIC}^{+}$, cell suspensions were sorted from BPH cell population.

\section{Xenograft studies}

Five-week-old male BALB/c (nu/nu) athymic mice were purchased from The Jackson Laboratory and housed in the University of Louisville vivarium under pathogen-free conditions. All experimental animals were maintained in accordance with the Institutional Animal Care and Use Committee approval and approval was obtained from the ethical committee of the University of Louisville, KY. The mice were randomly divided into four groups of eight each (a unique identification number was assigned to the animals, the numbers were drawn randomly and animals were logically assigned to different groups). At 7 weeks of age, the mice were subcutaneously injected with BPH1, CTBPH1, $\mathrm{ZIC}^{+}$, and $\mathrm{ZIC}^{-}$cells $\left(1 \times 10^{6}\right)$ in $50 \mu \mathrm{l}$ Matrigel (Corning). The mice were monitored twice weekly, and the tumor volumes were measured once per week. At the end of experiments, the mice were euthanized via $\mathrm{CO}_{2}$ asphyxiation; the tumors were removed and fixed in $10 \%$ formalin for histopathological studies.

\section{Statistical analysis}

All statistical analysis used GraphPad Prism 8.0a software (GraphPad Software, Inc., La Jolla, CA). An unpaired two-tailed Student's $t$-test was performed for two-group comparisons and one-way analysis of variance analysis was performed for multiple group comparisons. The statistical significance was set at a $P<0.05$, and values were presented as means $\pm \mathrm{SD}$.

\section{Results \\ Acute $\mathrm{Cd}$ exposure promotes Shh/ZIC2 expression in BPH1 cells}

We investigated whether acute $\mathrm{Cd}$ exposure impacted $\mathrm{BPH} 1$ cell viability. Although, growth inhibition of the Cdexposed BPH1 cells was seen, this result was not statistically significant versus vehicle-treated cells (Fig. 1a). Subsequent examination of prosurvival and proapoptotic proteins using western blots revealed that $\mathrm{Cd}$ exposure increased the expression of survival markers, such as $\mathrm{p} 65, \mathrm{Bcl} 2, \mathrm{Bcl}_{\mathrm{XL}}$, and XIAP but did not significantly alter the expression of proapoptotic proteins cleaved caspase-3 and PARP (Fig. 1b).

Next, a global RNA-sequence analysis was performed to determine the molecular events in Cd-exposed BPH1 cells. The results indicated that the induction of ZIC2 and Shh signaling appeared to be a prominent event in the transformation of Cd-exposed BPH cells (data not shown). Western blots and qRT-PCR confirmed that ZIC2 and Shh pathway markers were upregulated, validating our transcriptomic analysis. The results confirmed the activation of Shh signaling in Cd-exposed BPH1 cells, as determined by a decrease in PTCH1 expression and corresponding upregulation of Shh, GLI1, and ZIC2 (Fig. 1c). While Cd exposure induced the expression of GLI1, no significant alterations were noted in expressions of other GLI family members (GLI2 and GLI3) in the BPH1 cells (data not shown). Stem cell renewal is a function of ZIC2 and further confirms its activation following $\mathrm{Cd}$ exposure; thus, we also examined the expression of stem cell markers in Cd-exposed BPH1 cells. The results showed increased expression of Nanog, SOX2, CD44, and Notch1 confirming that $\mathrm{ZIC} 2$ activation may be a key response to $\mathrm{Cd}$ exposure in BPH1 cells (Fig. 1d). 


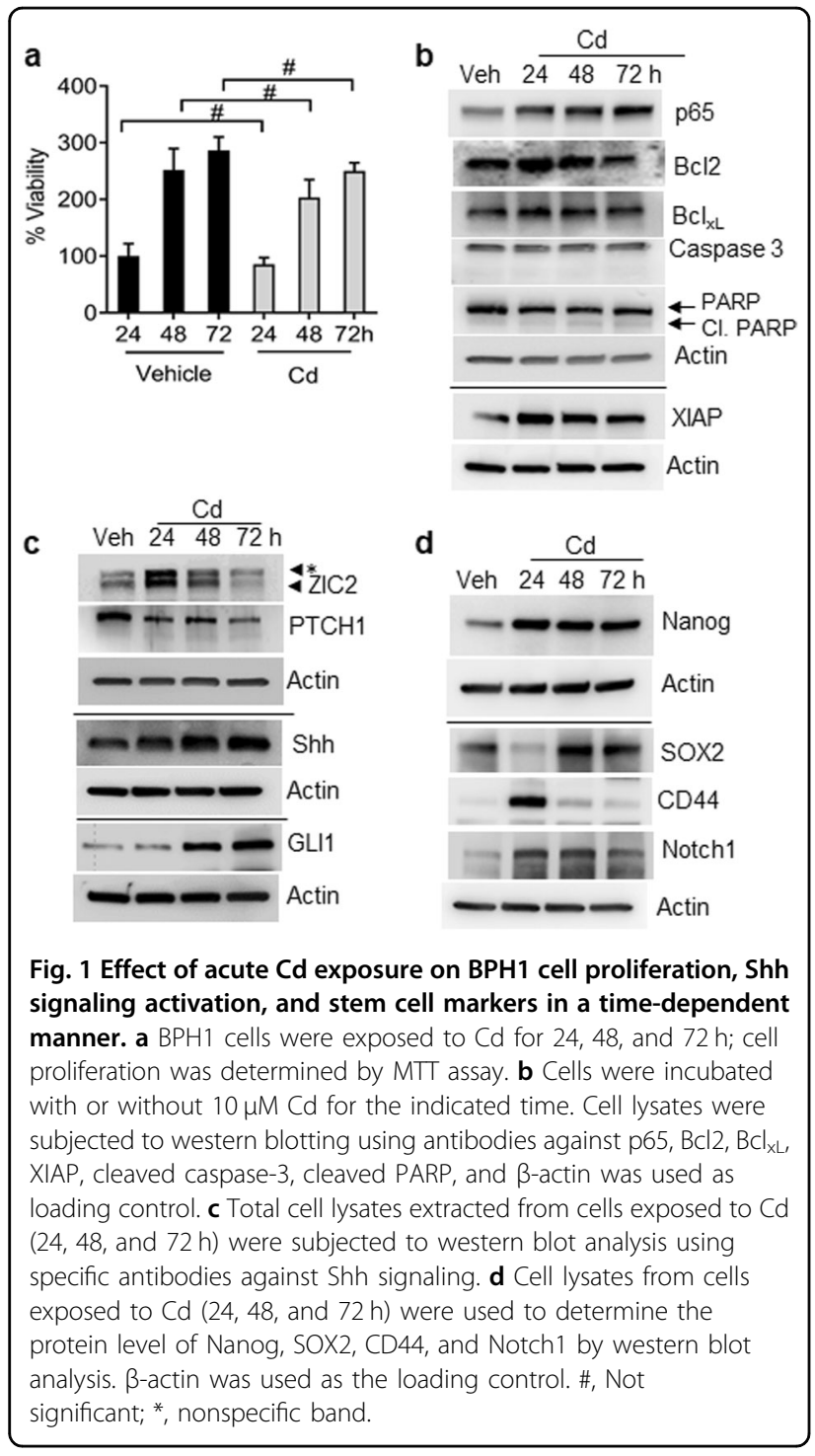

To further confirm that this activation is specific to $\mathrm{Cd}$ exposed BPH1 cells, we assessed ZIC2 levels in RWPE-1 cells following acute $\mathrm{Cd}$ exposure. No changes in ZIC2 mRNA and protein expression were noted in Cd-exposed RWPE-1 cells (Fig. 2a, b), suggesting that $\mathrm{Cd}$ activation of ZIC2 was specific to BPH1 cells.

Although the use of immortalized cell lines is imperative for metal carcinogenesis especially for transformation assay, the effect of the immortalization process itself on the molecular signature of the metal carcinogen-induced transformation remains a concern for researchers. To address this, we compared effect on cell viability and ZIC2/GLI1 expression on primary human BPH cells with normal primary $\mathrm{BPH}$ cells following $\mathrm{Cd}$ exposure. The $\mathrm{Cd}$ exposure reduced the cell viability (not significantly) as seen in primary human $\mathrm{BPH}$ cells and increased the expression of ZIC2 and GLI1 (Fig. 2c, d). This confirmed

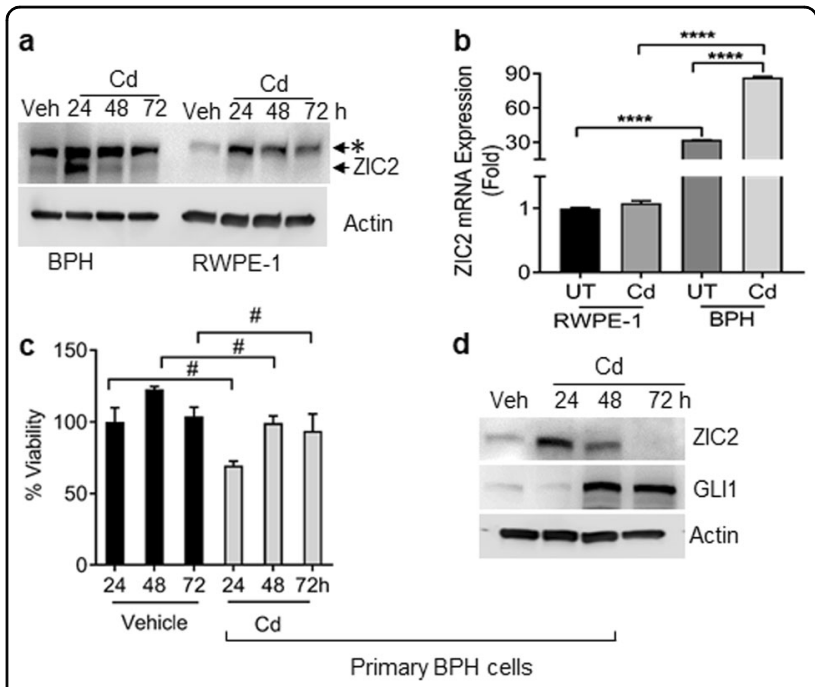

Fig. 2 Acute exposure of $\mathrm{Cd}$ induces ZIC2 in primary human BPH cells and BPH1 cells. a Cells were incubated with $10 \mu \mathrm{M} \mathrm{Cd}$ for the indicated time periods. Western blot analyses comparing ZIC2 expression in BPH1 cells (left four lanes) and RWPE-1 (right four lanes). b mRNA expression of ZIC2 in Cd-treated RWPE-1 and BPH1 cells. c Primary human BPH cells were exposed to $\mathrm{Cd}$ for indicated time points, MTT assay was performed to analyze the effect on cell viability. d Cell lysates from primary human BPH cells exposed to $\mathrm{Cd}(24,48$, and $72 \mathrm{~h}$ ) were used for western blot analysis using specific antibodies against ZIC2 and GLI1. $\beta$-actin was used as the loading control. ${ }^{* * *} p<0.0001$ and \#, not significant; ${ }^{*}$, nonspecific band.

that the molecular signature of $\mathrm{Cd}$ is not compromised in immortalized BPH1 cells.

\section{Chronic exposure to $\mathrm{Cd}$ induces malignant transformation of BPH1 cells}

In order to develop an in vitro model of Cd-induced malignant transformation, we chronically exposed $\mathrm{BPH} 1$ cells to $10 \mu \mathrm{M} \mathrm{Cd}$ for up to 16 months. We periodically performed anchorage-independent growth assays to determine the malignant transformation of $\mathrm{Cd}$-exposed cells. BPH1 cells began forming colonies after 6 months of $\mathrm{Cd}$ exposure, and the number of the colonies increased with exposure time. This confirmed that $\mathrm{Cd}$ exposure induced the malignant transformation of BPH1 cells (Fig. 3a).

We next performed western blot analysis to confirm whether the molecular signature of chronic $\mathrm{Cd}$ exposure was similar to acute exposure in the BPH1 cells. The results showed that similar to acute exposure, chronic $\mathrm{Cd}$ exposure also increased the expression of ZIC2, Shh, and GLI1. Upregulation of prosurvival proteins p65, XIAP, and Bcl2 stem cell markers Nanog and CD44 was also observed (Fig. 3b, c). Interestingly, we observed a timedependent change in the expression of EMT markers and $\beta$-catenin in the chronic Cd-exposed BPH1 cells with the upregulation of $\mathrm{N}$-cadherin, $\beta$-catenin, and downregulation of E-cadherin (Fig. 3c). To corroborate the 
a

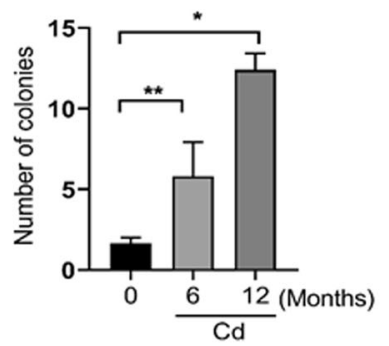

C

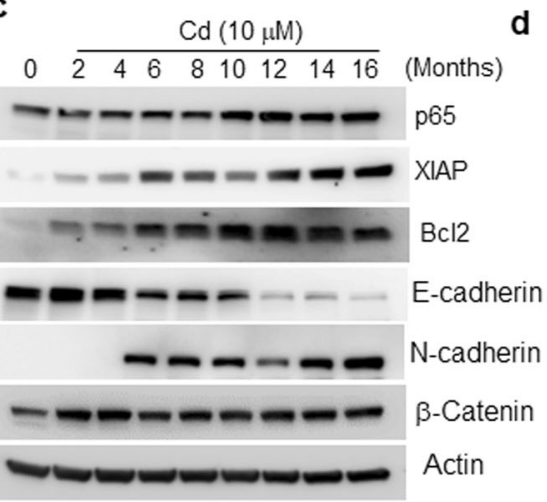

b $\frac{\mathrm{Cd}(10 \mu \mathrm{M})}{2-4(0-12)}$

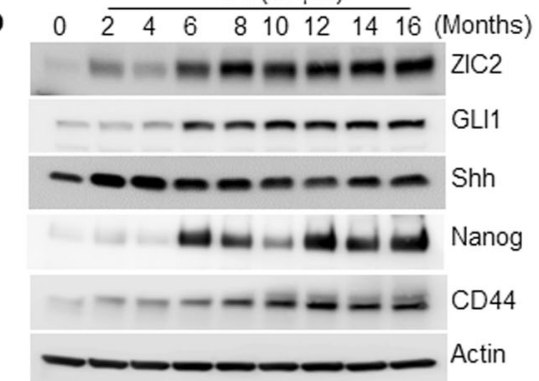

d

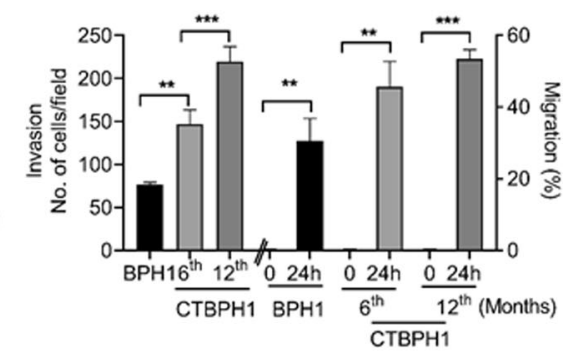

Fig. 3 Effect of chronic exposure to Cd on soft agar assay, shh signaling, stem cell properties, cell survival, and EMT marker's expression in CTBPH1 cells. a Soft agar assay for colony formation in Cd-treated BPH1 cells after 6 and 12 months. b Total cell lysate extracted from BPH1 cells exposed to $\mathrm{Cd}(10 \mu \mathrm{M})$ up to 16 months was used to immunoblot ZIC2, GLI1, Shh, Nanog, and CD44, c p65, XIAP, BCl2, EMT markers, and $\beta$-actin was used as a loading control. $\mathbf{d}$ A migration assay and Boyden chamber invasion assay were used to assess cell migration and invasion ability of CTBPH1 cells. ${ }^{* *} p<0.01$ and ${ }^{* *} p<0.001$.

western blot results further, we also performed cell invasion and migration assays in Cd-transformed cells. The results demonstrated that chronic $\mathrm{Cd}$ exposure significantly increased the invasive and migratory properties of cells exposed cells for 6 and 12 months versus vehicletreated control cells (Fig. 3d). These results collectively indicate that $\mathrm{Cd}$ exposure significantly induces the invasion and migration abilities of transformed cells.

Although, the BPH1 cells were chronically exposed to Cd up to 16 months to induce malignant transformation, we did not observe any significant phenotypic changes in the cells exposed beyond 12 months. Thus, we considered the cells exposed to $\mathrm{Cd}$ for 12 months to be completely transformed and will refer to them as СТВPH1 below.

\section{ZIC2 promotes CSC characteristics and EMT phenotype in malignant transformed BPH1 cells}

Self-renewal, higher tumorigenicity, sphere formation, and metastatic ability are characteristic properties of $\mathrm{CSCs}^{29,30}$. We used FACS to sort the ZIC2-negative and -positive cell populations at specific time points $(0,2,6,10$, and 12 months) to determine whether Cd-mediated ZIC2 CSCs properties in the transformed BPH1 cells. A gradual increase in the percentage of $\mathrm{ZIC}^{+}$cells was observed in the transforming cells; the 12-month timepoint had the highest fraction of positive cells (45-50\%; Fig. 4a). Interestingly, subsequent spheroid assays demonstrated that only $\mathrm{ZIC}^{+}$cells formed spheres while $\mathrm{ZIC}^{-}$cells did not form spheroids (Fig. 4b), confirming that ZIC2 expression was associated with the self-renewal properties of $\mathrm{Cd}$ exposed cells. This was corroborated via xenotransplantation experiments that showed only nude mice injected with $\mathrm{Cd}$-exposed $\mathrm{ZIC2}{ }^{+}$developed tumors versus mice injected with $\mathrm{ZIC}^{-}$cells (Fig. 4c). To further confirm that ZIC2 is a stem cell driver, we performed sphere formation assay in ZIC2 overexpressed in normal epithelial cells (RWPE-1). As seen in (Fig. 4d) ZIC2 overexpression in normal prostate epithelial cells formed spheroids and no spheroids were seen in control vector transfected cells. We next evaluated the expression of epithelial (E-cadherin), mesenchymal ( $\beta$-catenin), in $\mathrm{ZIC}^{+}$and $\mathrm{ZIC}^{-}$ CTBPH1 cells. Increased, MMP 9, slug and $\beta$-catenin expression followed by downregulation of E-cadherin was noted in the $\mathrm{ZIC}^{+}$cells as compared with $\mathrm{ZIC}^{-}$ CTBPH1 cells. (Fig. 4e).

Furthermore, invasion and migration assays demonstrated that $\mathrm{ZIC}^{+}$cells showed increased invasive and migratory properties (Fig. 4f) versus $\mathrm{ZIC2}^{-}$cells. These 

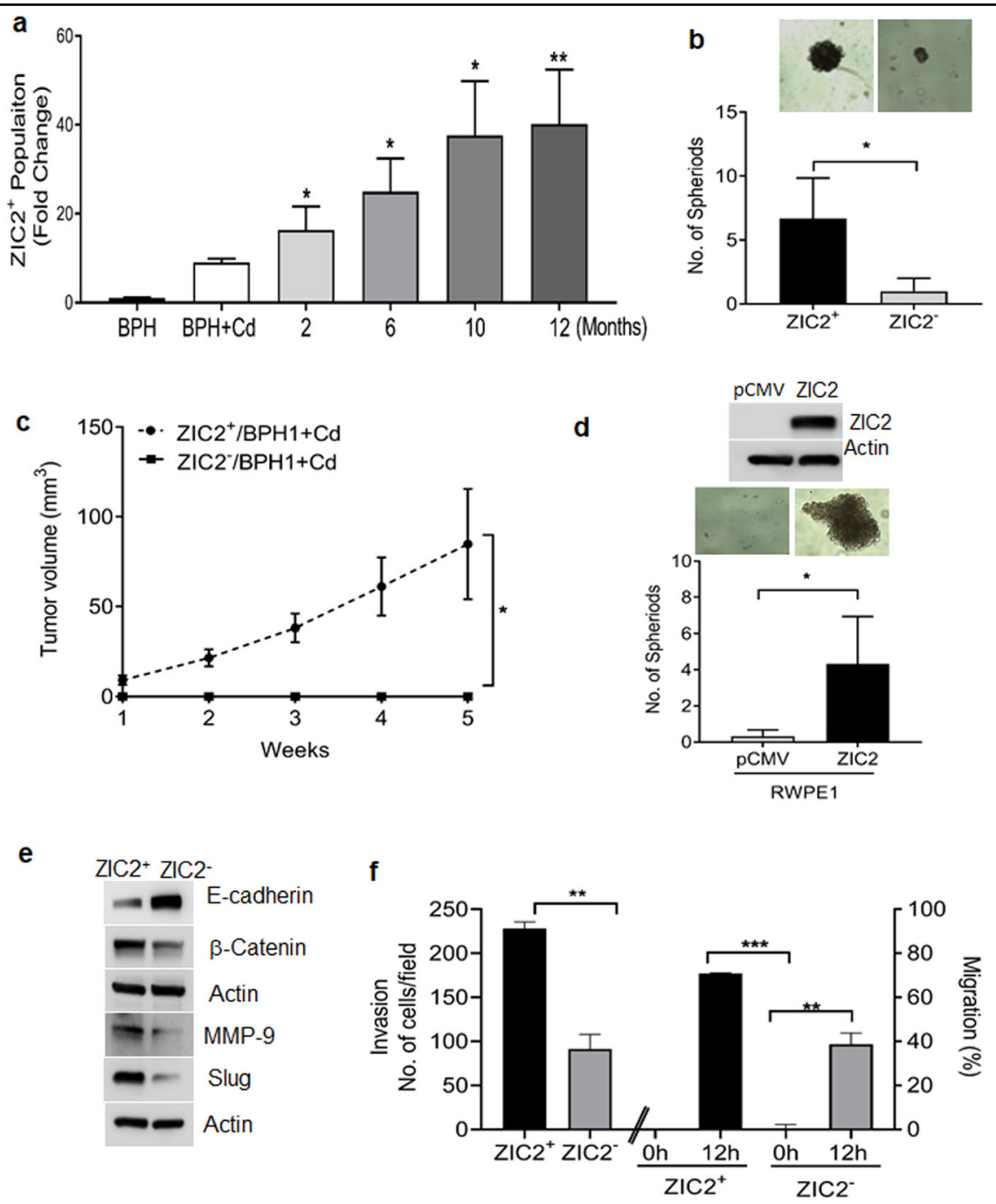

Fig. $4 \mathrm{ZIC2}^{+}$cancer cells have properties of CSCs. a Fluorescence-activated cell sorting (FACS) analysis of BPH1 cells exposed to $10 \mu \mathrm{M}$ Cd $(0,2,6$, 10, and 12 months) expressing ZIC2. b Spheroid formation assay for $\mathrm{ZIC2}^{+}$and $\mathrm{ZIC2}^{-}$cell population harvested from FACS. c Tumor burden in mice xenotransplanted with $\mathrm{ZIC2}^{+}$and $\mathrm{ZIC2}{ }^{-}$(cells furthest from $\mathrm{ZIC2}^{+}$gating) was analyzed during 5 weeks of study. $\mathbf{d}$ Spheroid formation assay for RWPE1 cells overexpressing ZIC2. e Cell lysates from $\mathrm{ZIC2}^{+}$and $\mathrm{ZIC2}^{-}$were subjected to western blotting using antibodies against E-Cadherin, $\beta$-catenin, slug, and MMP 9. $\beta$-actin was used as the loading control. f Migration assay and Boyden chamber invasion assay were used to assess cell migration and invasion ability of $\mathrm{ZIC2}^{+}$and $\mathrm{ZIC2}^{-}$cells. ${ }^{* *} p<0.01$ and ${ }^{* * *} p<0.001$.

results suggest that ZIC2 may aid in the transformation of Cd-exposed BPH1 by inducing mesenchymal phenotype and promoting stem cell renewal.

\section{Silencing ZIC2 abolishes self-renewal potency of CTBPH1 cells}

Considering that $\mathrm{Cd}$-mediated $\mathrm{ZIC} 2$ activation promoted the self-renewal properties of the CTBPH1 cells, we inhibited the ZIC2 expression in these cells to determine if this altered their self-renewal potency. Spheroid assays showed that silencing ZIC2 expression in CTBPH1 cells completely abrogated the formation of spheroids versus cells transfected with scrambled vectors
(Fig. 5a). Subsequent western blots and RT-qPCR assays revealed that silencing $\mathrm{ZIC} 2$ expression caused the inhibition of stem markers SOX2, Notch1, and CD44 expression, suggesting that ZIC2 may be a master regulator of stem cell markers (Fig. 5b, c). Interestingly, cell viability assays showed that inhibition of ZIC2 did not inhibit the growth of CTBPH1 cells (Fig. 5d) nor alter the expression of prosurvival ( $\mathrm{p} 65)$ or proapoptotic (PARP cleavage) markers. Additionally, inhibition of ZIC2 failed to alter the levels of GLI1 levels in the CTBPH1 cells (Fig. 5e), suggesting that ZIC2 activation is necessary for stem cell renewal but is not involved in the prosurvival or proapoptotic signaling during $\mathrm{Cd}$-mediated transformation. 

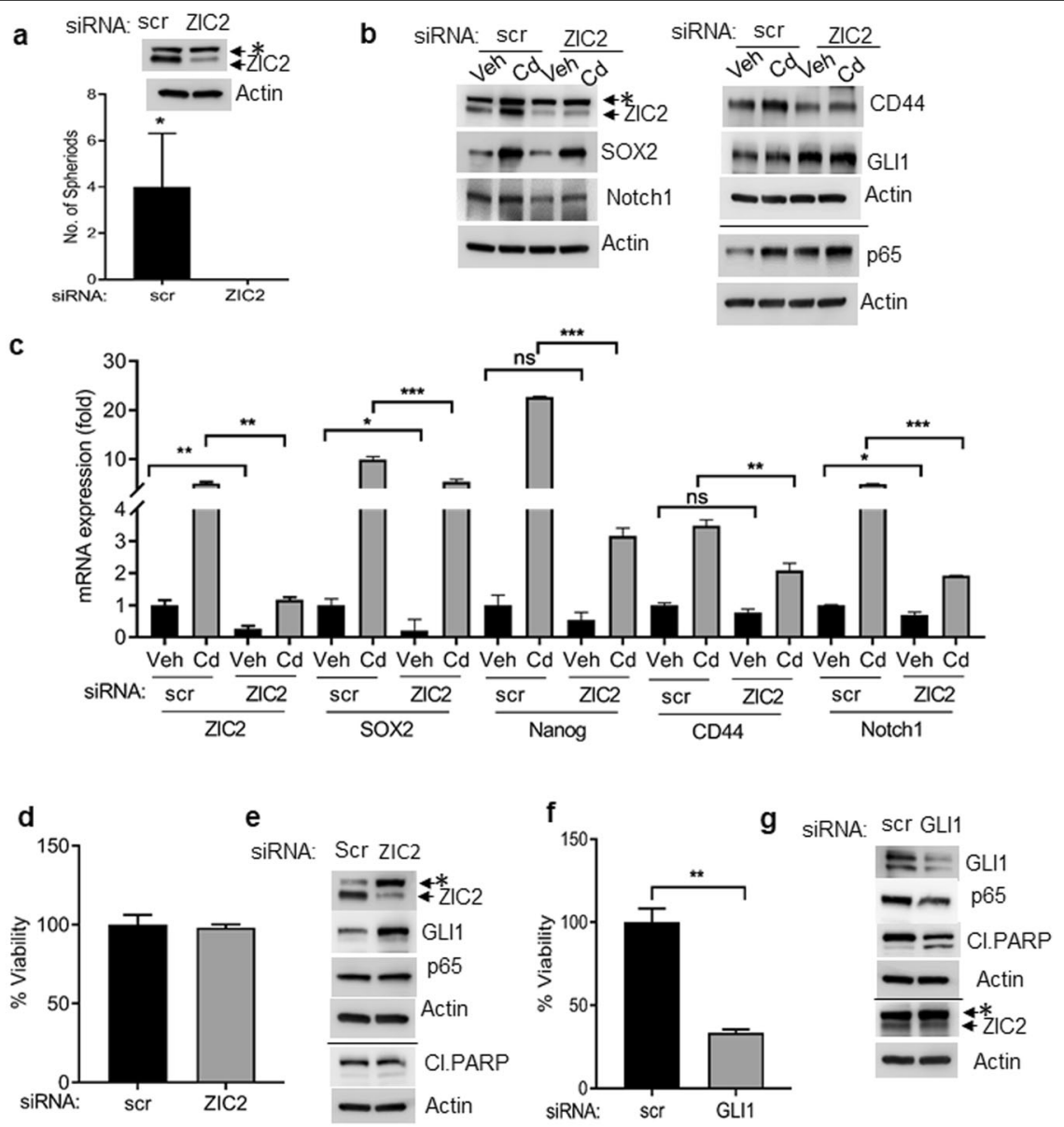

Fig. 5 Effect of ZIC2 and GLI1 siRNA knock down on viability, survival, and stem cell markers expression in CTBPH1 cells. a Spheroid formation assay for CTBPH1 cells transfected with scrambled (scr) and ZIC2 siRNA. b CTBPH1 cells transfected with scr or ZIC2 siRNA were incubated with or without $\mathrm{Cd}$ for $24 \mathrm{~h}$. Cell lysates were subjected to western blotting using antibodies against ZIC2, GLI1, SOX2, CD44, p65, Notch1, and $\beta$-actin. c RNA isolated from CTBPH1 cells transfected with scr or ZIC2 siRNA and incubated with or without $\mathrm{Cd}$ for $24 \mathrm{~h}$ was subjected to qRT-PCR analysis using ZIC2, SOX2, Nanog, CD44, and Notch1 primers. d Cell viability of CTBPH1 cells treated with scr or ZIC2 siRNA was determined by trypan blue assay. e ZIC2, GLI1, p65, and cleaved PARP expression in cell lysates prepared from scr and ZIC2 siRNA-treated CTBPH1 cells. $\mathbf{f}$ Cell viability of CTBPH1 treated with scr or GLI1 siRNA: The cell viability was determined by trypan blue assay. $\mathbf{g}$ Cell lysates were prepared from scr and GLI1 siRNA-treated CTBPH1 cells to determine the protein levels of ZIC2, GLI1, p65, and cleaved PARP by western blot analysis. \#, not significant, ${ }^{*} p<0.05$, ${ }^{* *} p<0.01$, and ${ }^{* * *} p<0.001 ;{ }^{*}$, nonspecific band.

\section{GLI1 knock down decreases the proliferation of CTBPH1 cells}

As shown above, $\mathrm{Cd}$ exposure induced $\mathrm{ZIC2}$ - and Shhmediated GLI1 signaling in the CTBPH1 cells. We postulated that the induction of prosurvival or inhibition of proapoptotic signaling may be regulated by activation of GLI1. Interestingly, cell viability assays showed that inhibition of GLI1 inhibited the growth of CTBPH1 cells (Fig. 5f). To confirm this, we silenced GLI1 expression in CTBPH1 cells resulting in the upregulation of PARP and concurrent inhibition of p65 expression. Moreover, inhibition of GLI1 failed to alter the levels of ZIC2 levels in the CTBPH1 cells (Fig. 5g).

\section{Determination of the molecular interplay between ZIC2 and GLI1 activation by $\mathrm{Cd}$}

These results indicate that both $\mathrm{ZIC} 2$ and Shh/ GLI1 signaling may be necessary for Cd-induced transformation of BPH1 cells. To delineate how the GLI1 and ZIC2 molecularly interact and coordinate their functions during Cd-mediated transformation, we first investigated the localization of these two transcription factors in the CTBPH1 cells. Western blot and immunofluorescence analysis revealed that $\mathrm{Cd}$ exposure caused induction and nuclear accumulation of ZIC2 and GLI1 versus vehicletreated control cells (Fig. 6a, b). Previous studies have suggested that GLI1 physically interacts with ZIC2 

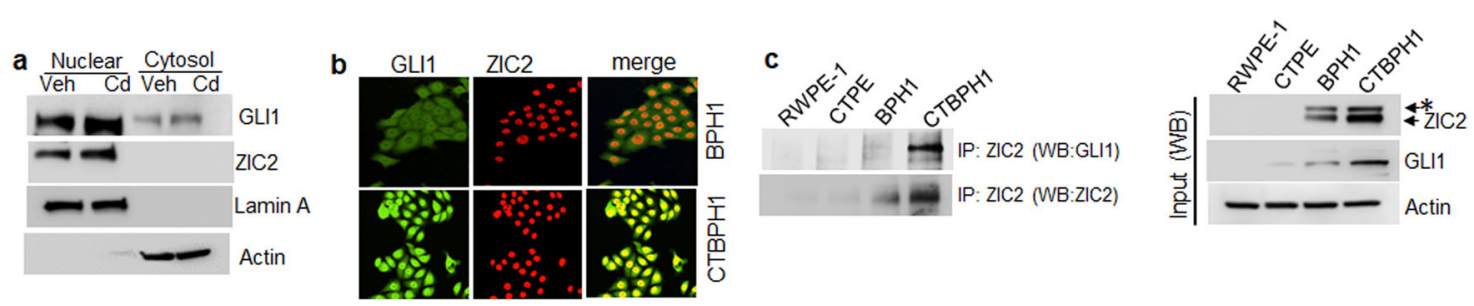

Fig. 6 Cd exposure leads to the nuclear translocation and retention of GLI1 and ZIC2. a Western blot analysis of cytosolic and nuclear fractions of BPH1 cells treated with $10 \mu \mathrm{M} \mathrm{Cd}$ for $24 \mathrm{~h}$. For loading controls, the same membranes were reprobed with antibodies to $\beta$-actin and lamin A for cytoplasmic and nuclear fractions, respectively. b Immunofluorescence staining patterns of ZIC2 and GLI1 in BPH1 and CTBPH1 cells. c Cell lysates of RWPE-1, CTPE, BPH1 and CTBPH1 cells were immunoprecipitated with anti-ZIC2 antibody. The immunoprecipitates were analyzed by western blot with anti-GLI1; ${ }^{*}$, nonspecific band.

protein via its zinc finger domain ${ }^{31}$; thus, we performed IPs to confirm whether Cd-induced BPH carcinogenesis occurs due to the interaction between these two proteins. Unlike the vehicle-treated BPH1 cells that showed a complete absence of GLI1/ZIC2 binding, strong binding was observed in CTBPH1 cells (Fig. 6c). These results suggest that GLI1/ZIC2 interaction plays an important role in the Cd-mediated transformation of BPH1 cells.

\section{Xenograft analysis of CTBPH1 cells}

Next, we investigated whether CTBPH1 cells could induce tumor formation in nude mice. We observed a progressive increase in tumor growth in mice injected with CTBPH1 cells versus mice injected with vehiclecontrol cells (Fig. 7a) over a 4-week period post inoculation. Additionally, consistent with the aforementioned in vitro findings, the tumors isolated from the CTBPH1injected mice demonstrated increased ZIC2, GLI1, and p65 expressions (Fig. 7b).

\section{ZIC2 expression in human BPH specimens}

The immunohistochemistry of $\mathrm{BPH}$ tissue microarrays comprising tissue sections from $80 \mathrm{BPH}$ patients showed that ZIC2 immunostaining was predominant in BPH specimens versus normal prostate tissue (Fig. 7c). Examination of the mRNA expression levels of ZIC2 in $\mathrm{BPH}$ specimens by qRT-PCR revealed a 20 -fold increase in expression in $\mathrm{BPH}$ tissue versus normal prostate tissue (Fig. 7d). A higher expression of ZIC2 in $\mathrm{BPH}$ specimens suggest that patients with $\mathrm{BPH}$ could be susceptible, if exposed $\mathrm{Cd}$ for potential malignant transformation.

\section{Discussion}

Although Cd is a well-known human prostatic carcinogen $^{32}$, the underlying mechanisms involved in Cd carcinogenesis remain unclear. Here, we demonstrated that long-term chronic exposure to $\mathrm{Cd}$ to $\mathrm{BPH} 1$ cells induced transcriptional changes responsible for stem cell renewal, proliferation, and tumorigenesis of the transformed CTBPH1 cells. Cd-mediated ZIC2 activation-initiated
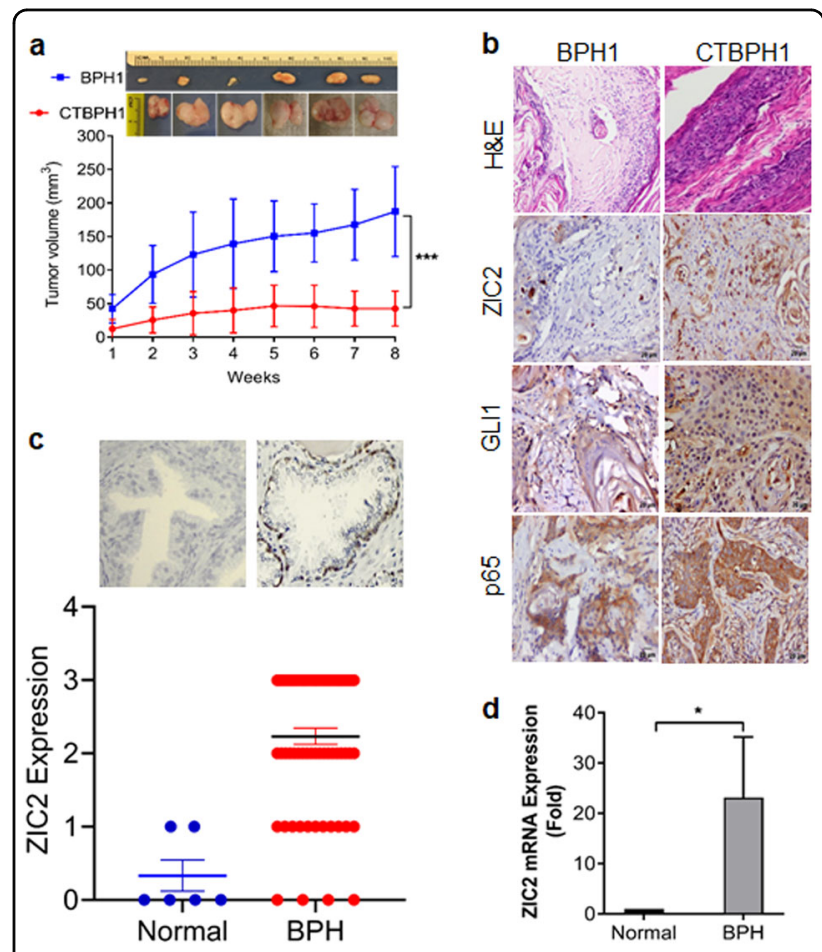

Fig. 7 CTBPH1 cells developed tumors in mice. The BPH1 cells exposed to $\mathrm{Cd}$ for 12 months were injected subcutaneously into nude mice. After 8 weeks, the mice were euthanized using $\mathrm{CO}_{2}$, and the tumor was isolated for further examination. a Representative image of excised tumors and graphical representation of the tumor volume in 8 weeks study period. $\mathbf{b}$ Immunohistochemical analysis of tissue sections derived from BPH1 and CTBPH1 cells for ZIC2, GLI1, and p65. c ZIC2 expression in normal human prostate and BPH specimens. d mRNA expression of ZIC2 in normal human prostate and BPH human specimens.

stem cell renewal and activation of GLI1: both of these steps promoted cell proliferation of the transformed cells. Thus, we confirmed that the dynamic interaction of ZIC2 and GLI1 are necessary for Cd-induced malignant transformation of $\mathrm{BPH}$ cells, a phenomenon not observed in normal prostate epithelial cells. 
We previously demonstrated the implantation of $\mathrm{Cd}$ transformed RWPE-1 cells; these cells formed tumors in xenograft models ${ }^{33,34}$. Similarly, this study helped show that chronic exposure to $\mathrm{Cd}$ resulted in malignant transformation of BPH1 cells. We previously showed that defective autophagy was responsible for Cd-induced transformation of RWPE-1 ${ }^{33,35}$. Here, ZIC2 and GLI1 is responsible for $\mathrm{Cd}$-inducted transformation in $\mathrm{BPH} 1$ cells, and it appears that it is specific to BPH1 cells and not to normal prostate epithelial cells.

Earlier studies established the oncogenic role of ZIC2 in pancreatic ductal adenocarcinoma cells by activating the expressions of FGFR3 and ANXA $8^{36}$. Similarly, the stem cell renewal function of ZIC2 was established ${ }^{37}$ by activating the stem cell transcription factors, such as Oct4, Sox2, and Nanog as binding sites ${ }^{38}$. We also found that Nanog and CD44 were upregulated in CTBPH1 cells as compared with vehicle-treated cells. Previous studies demonstrated that CSCs may be responsible for tumor initiation, invasion, distant metastasis, chemo-resistance, self-renewal, and differentiation potential ${ }^{39}$. Thus, we set out to isolate $\mathrm{ZIC}^{+}$cells from CTBPH1 cells and characterize the ZIC2 function. CSCs are believed to be able to form spheres in culture that can then exhibit extensive similarities to endogenous CSCs in human tumor tissues $^{40}$. Here, sphere formation assays using $\mathrm{ZIC}^{+}$and $\mathrm{ZIC}^{-}$cells revealed the increased ability of $\mathrm{ZIC}^{+}$cell to form spheroids, suggesting that ZIC2 activation imparts CSC properties to Cd-exposed BPH1 cells. Interestingly, upon testing the in vivo potency of $\mathrm{ZIC2}^{+}$versus $\mathrm{ZIC2}^{-}$ cells in athymic mice, we observed tumor formation in mice injected with $\mathrm{ZIC}^{+}$cells but not in those injected with $\mathrm{ZIC2}{ }^{-}$cells. However, our results also demonstrated that the inhibition of ZIC2 expression did alter the expression of cell survival and proapoptotic markers, suggesting activation of an alternative machinery in $\mathrm{Cd}$ exposed cells.

Recently Chan et al. ${ }^{14}$ demonstrated that the overexpression of ZIC2-induced nuclear retention of GLI1: the downstream effector of oncogenic Shh signaling. Other studies have suggested that GLI1 physically interacts with ZIC2 protein via a zinc finger domain ${ }^{31,41,42}$. We found that Cd-induced Shh ligands that in turn inhibited PTCH1 expression leading to the activation of GLI1 and downstream prosurvival targets, such as $\mathrm{Bcl} 2, \mathrm{Bcl}_{\mathrm{XL}}$, and XIAP. This strongly suggested that activation of Shh signaling initiated the prosurvival machinery and promotion of cell proliferation in Cd-exposed BPH1 cells; this was later corroborated when we found that silencing GLI1 expression inhibited cell proliferation in CTBPH1 cells. Interestingly, we also noted that the interaction of ZIC2 and GLI1 was stronger in CTBPH1 cells than RWPE-1 and BPH1 cells. This suggests that the oncogenic effects of ZIC2 could be due to the interaction between these two proteins and could in turn aid in the tumorgenicity of the CTBPH1 cells.

Recent studies have demonstrated that Shh activation plays a central role in EMT regulation, involving the loss of cell-cell adhesion, changes in cell morphology, and the propensity to migrate and invade $\mathrm{CaP}^{43,44}$. Wei and Shaikh ${ }^{45}$ reported that prolonged $\mathrm{Cd}$ treatment in triplenegative breast cancer cells stimulates cell proliferation, adhesion, cytoskeleton reorganization, as well as migration and invasion. Here, we observed that both $\mathrm{ZIC}^{+}$cells and chronic exposure to $\mathrm{Cd}$-induced expression of mesenchymal marker, $\beta$-catenin. The $\mathrm{Wnt} / \beta$-catenin pathway plays a pivotal role in multiple malignancies, including regulating cell proliferation, EMT, and migratory process ${ }^{46,47}$. Moreover, Wnt/ $\beta$-catenin signaling could also regulate Zic gene expression $^{48}$. Thus, our results strongly suggest that chronic $\mathrm{Cd}$ exposure promotes invasion, migration, and EMT in $\mathrm{BPH} 1$ cells via activation of the $\mathrm{Wnt} / \beta$-catenin signaling pathway.

In summary, we found that both exposure of $\mathrm{Cd}$ in BPH1 cells causes malignant transformation and tumorigenesis via upregulation of Shh/GLI signaling, as well as ZIC2 activation. We also found that both ZIC2 and GLI1 function as complementary signals initiating stem cell renewal and leads invasion as well as migration, respectively. Thus, they are necessary components for $\mathrm{Cd}$ induced prostate carcinogenesis.

\section{Acknowledgements \\ The work was supported by NIEHS-R01ES028102, R01ES030019 to C.D., and R01ES027778 to J.C.S.}

\section{Conflict of interest}

The authors declare that the content has not been published or submitted for publication elsewhere. Further, all authors have significantly contributed to the work and agree with the content of the manuscript. The authors declare that they have no conflict of interests.

\section{Publisher's note}

Springer Nature remains neutral with regard to jurisdictional claims in published maps and institutional affiliations.

Received: 4 November 2019 Revised: 23 January 2020 Accepted: 28 January 2020

Published online: 17 February 2020

\footnotetext{
References

1. Deng, L. et al. Evaluation of the therapeutic effect against benign prostatic hyperplasia and the active constituents from Epilobium angustifolium L. J. Ethnopharmacol. 232, 1-10 (2019).

2. Sarbishegi, M., Khani, M., Salimi, S., Valizadeh, M. \& Sargolzaei Aval, F. Antiproliferative and antioxidant effects of withania coagulans extract on benign prostatic hyperplasia in rats. Nephrourol. Mon. 8, e33180 (2016).

3. Bostwick, D. G. et al. The association of benign prostatic hyperplasia and cancer of the prostate. Cancer 70, 291-301 (1992).

4. Miah, S. \& Catto, J. BPH and prostate cancer risk. Indian J. Urol. 30, 214-218 (2014).

5. Ornstein, D. K., Rao, G. S., Smith, D. S. \& Andriole, G. L. The impact of systematic prostate biopsy on prostate cancer incidence in men with symptomatic
} 
benign prostatic hyperplasia undergoing transurethral resection of the prostate. J. Urol. 157, 880-883 (1997).

6. Armenian, H. K., Lilienfeld, A. M., Diamond, E. L. \& Bross, I. D. Relation between benign prostatic hyperplasia and cancer of the prostate. A prospective and retrospective study. Lancet 2, 115-117 (1974).

7. Dai, X., Fang, X., Ma, Y. \& Xianyu, J. Benign prostatic hyperplasia and the risk of prostate cancer and bladder cancer: a meta-analysis of observational studies. Med. (Baltim.) 95, e3493 (2016).

8. Tchounwou, P. B., Yedjou, C. G., Patlolla, A. K. \& Sutton, D. J. Heavy metal toxicity and the environment. Exp. Suppl. 101, 133-164 (2012).

9. Zhang, L., Zhu, Y., Hao, R., Shao, M. \& Luo, Y. Cadmium levels in tissue and plasma as a risk factor for prostate carcinoma: a meta-analysis. Biol. Trace Elem. Res. 172, 86-92 (2016).

10. Siewit, C. L., Gengler, B., Vegas, E., Puckett, R. \& Louie, M. C. Cadmium promotes breast cancer cell proliferation by potentiating the interaction between ERalpha and c-Jun. Mol. Endocrinol. 24, 981-992 (2010).

11. Akesson, A., Julin, B. \& Wolk, A. Long-term dietary cadmium intake and postmenopausal endometrial cancer incidence: a population-based prospective cohort study. Cancer Res. 68, 6435-6441 (2008).

12. Benbrahim-Tallaa, L. et al. Cadmium malignantly transforms normal human breast epithelial cells into a basal-like phenotype. Environ. Health Perspect. 117, 1847-1852 (2009).

13. Merrick, B. A. et al. Arsenite malignantly transforms human prostate epithelial cells in vitro by gene amplification of mutated KRAS. PLOS ONE 14, e0215504 (2019).

14. Chan, D. W. et al. Zic2 synergistically enhances Hedgehog signalling through nuclear retention of Gli1 in cervical cancer cells. J. Pathol. 225, 525-534 (2011).

15. Sabater, L. et al. ZIC antibodies in paraneoplastic cerebellar degeneration and small cell lung cancer. J. Neuroimmunol. 201-202, 163-165 (2008).

16. Sakuma, K. et al. Expression status of Zic family member 2 as a prognostic marker for oral squamous cell carcinoma. J. Cancer Res. Clin. Oncol. 136, 553-559 (2010).

17. Aruga, J. et al. The mouse zic gene family. Homologues of the Drosophila pairrule gene odd-paired. J. Biol. Chem. 271, 1043-1047 (1996).

18. Mizugishi, K., Aruga, J., Nakata, K. \& Mikoshiba, K. Molecular properties of Zic proteins as transcriptional regulators and their relationship to GLI proteins. J. Biol. Chem. 276, 2180-2188 (2001).

19. Gonnissen, A., Isebaert, S. \& Haustermans, K. Hedgehog signaling in prostate cancer and its therapeutic implication. Int. J. Mol. Sci. 14, 13979-14007 (2013).

20. Ibuki, N. et al. TAK-441, a novel investigational smoothened antagonist, delays castration-resistant progression in prostate cancer by disrupting paracrine hedgehog signaling. Int. J. Cancer 133, 1955-1966 (2013).

21. Noubissi, F. K., Yedjou, C. G., Spiegelman, V. S. \& Tchounwou, P. B. Cross-talk between $\mathrm{Wnt}$ and $\mathrm{Hh}$ signaling pathways in the pathology of basal cell carcinoma. Int. J. Environ. Res. Public Health 15, E1442 (2018).

22. Wilson, C. W. \& Chuang, P. T. Mechanism and evolution of cytosolic Hedgehog signal transduction. Development 137, 2079-2094 (2010).

23. Katoh, Y. \& Katoh, M. Hedgehog target genes: mechanisms of carcinogenesis induced by aberrant hedgehog signaling activation. Curr. Mol. Med. 9, 873-886 (2009).

24. Chandrasekaran, B. et al. Molecular insights: suppression of EGFR and AKT activation by a small molecule in non-small cell lung cancer. Genes Cancer $\mathbf{8}$, 713-724 (2017).

25. Suman, S. et al. Activation of AKT signaling promotes epithelial-mesenchymal transition and tumor growth in colorectal cancer cells. Mol. Carcinog. 53(Suppl 1), E151-E160 (2014)
26. Das, T. P., Suman, S. \& Damodaran, C. Induction of reactive oxygen species generation inhibits epithelial-mesenchymal transition and promotes growth arrest in prostate cancer cells. Mol. Carcinog. 53, 537-547 (2014).

27. Tyagi, A. et al. Combination of androgen receptor inhibitor and cisplatin, an effective treatment strategy for urothelial carcinoma of the bladder. Urol. Oncol. 37 , 492-502 (2019)

28. Dahiya, N. R. et al. A natural molecule, urolithin A, downregulates androgen receptor activation and suppresses growth of prostate cancer. Mol. Carcinog. 57, 1332-1341 (2018).

29. Bleau, A. M. et al. Sphere-derived tumor cells exhibit impaired metastasis by a host-mediated quiescent phenotype. Oncotarget 6, 27288-27303 (2015).

30. Qiu, X. et al. Characterization of sphere-forming cells with stem-like properties from the small cell lung cancer cell line H446. Cancer Lett. 323, 161-170 (2012).

31. Koyabu, Y., Nakata, K., Mizugishi, K., Aruga, J. \& Mikoshiba, K. Physical and functional interactions between Zic and Gli proteins. J. Biol. Chem. 276 6889-6892 (2001).

32. Huff, J., Lunn, R. M., Waalkes, M. P., Tomatis, L. \& Infante, P. F. Cadmium-induced cancers in animals and in humans. Int. J. Occup. Environ. Health 13, 202-212 (2007).

33. Kolluru $V_{\text {., }}$ et al (2017). Induction of Plac8 promotes pro-survival function of autophagy in cadmium-induced prostate carcinogenesis. Cancer Lett. 408, 121-129.

34. Pal, D. et al. Inhibition of autophagy prevents cadmium-induced prostate carcinogenesis. Br. J. Cancer 117, 56-64 (2017).

35. Kolluru, V., Tyagi, A., Chandrasekaran, B., Ankem, M. \& Damodaran, C. Induction of endoplasmic reticulum stress might be responsible for defective autophagy in cadmium-induced prostate carcinogenesis. Toxicol. Appl. Pharm. 373, 62-68 (2019).

36. Inaguma, S., Ito, H., Riku, M., Ikeda, H. \& Kasai, K. Addiction of pancreatic cancer cells to zinc-finger transcription factor ZIC2. Oncotarget 6, 28257-28268 (2015).

37. Zhu, P. et al. ZIC2-dependent OCT4 activation drives self-renewal of human liver cancer stem cells. J. Clin. Invest. 125, 3795-3808 (2015).

38. Lim, L. S. et al. Zic3 is required for maintenance of pluripotency in embryonic stem cells. Mol. Biol. Cell 18, 1348-1358 (2007).

39. $\mathrm{Hu}$, J. et al. BTF3 sustains cancer stem-like phenotype of prostate cancer via stabilization of BMI1. J. Exp. Clin. Cancer Res. 38, 227 (2019).

40. Vermeulen, L., Sprick, M. R., Kemper, K., Stassi, G. \& Medema, J. P. Cancer stem cells-old concepts, new insights. Cell Death Differ. 15, 947-958 (2008).

41. Merzdorf, C. S. Emerging roles for zic genes in early development. Dev. Dyn. 236, 922-940 (2007)

42. Wang, K, Pan, L., Che, X., Cui, D. \& Li, C. Gli1 inhibition induces cell-cycle arrest and enhanced apoptosis in brain glioma cell lines. J. Neurooncol. 98, 319-327 (2010).

43. Koeneman, K. S., Yeung, F. \& Chung, L. W. Osteomimetic properties of prostate cancer cells: a hypothesis supporting the predilection of prostate cancer metastasis and growth in the bone environment. Prostate 39, 246-261 (1999).

44. Yamamichi, F. et al. Sonic hedgehog and androgen signaling in tumor and stromal compartments drives epithelial-mesenchymal transition in prostate cancer. Scand. J. Urol. 48, 523-532 (2014).

45. Wei, Z. \& Shaikh, Z. A. Cadmium stimulates metastasis-associated phenotype in triple-negative breast cancer cells through integrin and beta-catenin signaling. Toxicol. Appl. Pharm. 328, 70-80 (2017).

46. Anastas, J. N. \& Moon, R. T. WNT signalling pathways as therapeutic targets in cancer. Nat. Rev. Cancer 13, 11-26 (2013).

47. Jeong, W. J. et al. Ras stabilization through aberrant activation of Wnt/betacatenin signaling promotes intestinal tumorigenesis. Sci. Signal 5, ra30 (2012).

48. Nyholm, M. K., Wu, S. F., Dorsky, R. I. \& Grinblat, Y. The zebrafish zic2a-zic5 gene pair acts downstream of canonical Wnt signaling to control cell proliferation in the developing tectum. Development 134, 735-746 (2007). 